PROCEEDINGS OF THE

AMERICAN MATHEMATICAL SOCIETY

Volume 129, Number 12, Pages 3631-3636

S 0002-9939(01)05982-2

Article electronically published on April 25, 2001

\title{
CONFORMAL DIMENSION OF THE ANTENNA SET
}

\author{
CHRISTOPHER J. BISHOP AND JEREMY T. TYSON
}

(Communicated by Albert Baernstein II)

\begin{abstract}
We show that the self-similar set known as the "antenna set" has the property that $\inf _{f} \operatorname{dim}(f(X))=1$ (where the infimum is over all quasiconformal mappings of the plane), but that this infimum is not attained by any quasiconformal map; indeed, is not attained for any quasisymmetric map into any metric space.
\end{abstract}

Given a compact metric space $X$ we define its conformal dimension as

$$
\operatorname{Cdim}(X)=\inf _{f} \operatorname{dim}(f(X)),
$$

where the infimum is over all quasisymmetric maps of $X$ into some metric space and "dim" denotes Hausdorff dimension. Recall that $f$ is quasisymmetric if there is a homeomorphism $\eta$ of $[0, \infty)$ to itself such that

$$
|x-y| \leq t|x-z| \Rightarrow|f(x)-f(y)| \leq \eta(t)|f(x)-f(z)| .
$$

For $f: \mathbb{R}^{n} \rightarrow \mathbb{R}^{n}, n \geq 2$, these are exactly the same as quasiconformal maps. This notion of conformal dimension was introduced by Pansu [9. See also [4] and [10]. In this note we are interested in the question of whether the infimum above must be attained by some quasisymmetric image of $X$. It is known that for some sets (e.g., the usual Cantor middle thirds set in $\mathbb{R}$ ) the conformal dimension is zero, whereas any quasisymmetric image must have strictly positive dimension. Here we answer a question of Heinonen's (Section 15, 7]) by proving

Theorem 1. There is a compact, connected $X \subset \mathbb{R}^{2}$ so that for every quasisymmetric map $f$ of $X$ into a metric space, $\operatorname{dim}(f(X))>1=\operatorname{Cdim}(X)$.

In particular, this holds for all quasiconformal self-maps of the plane, which is already an interesting case. Our set is the self-similar set associated to the four conformal contractions

$$
\begin{gathered}
f_{1}(z)=\frac{1}{2} z, \quad f_{2}(z)=\frac{1}{2} z+\frac{1}{2}, \\
f_{3}^{\alpha}(z)=\alpha i z+\frac{1}{2}, \quad f_{4}^{\alpha}(z)=-\alpha i z+\frac{1}{2}+\alpha i,
\end{gathered}
$$

Received by the editors November 15, 1999 and, in revised form, April 27, 2000.

2000 Mathematics Subject Classification. Primary 30C62; Secondary 28A78.

Key words and phrases. Quasiconformal map, Hausdorff dimension, conformal dimension, selfsimilar sets.

The first author was partially supported by NSF Grant DMS 98-00924. The second author was partially supported by an NSF postdoctoral fellowship. 
where $0<\alpha<\frac{1}{2}$. For $E \subset \mathbb{R}^{2}$, let

$$
F_{\alpha}(E)=f_{1}(E) \cup f_{2}(E) \cup f_{3}^{\alpha}(E) \cup f_{4}^{\alpha}(E),
$$

and let $X_{\alpha}$ be the unique compact set such that $X=F_{\alpha}(X)$ (see Section 9.1 of [5] for the existence and uniqueness of this set). The set $X_{\alpha}$ is shown in Figure 1 for a few different values of $\alpha$.

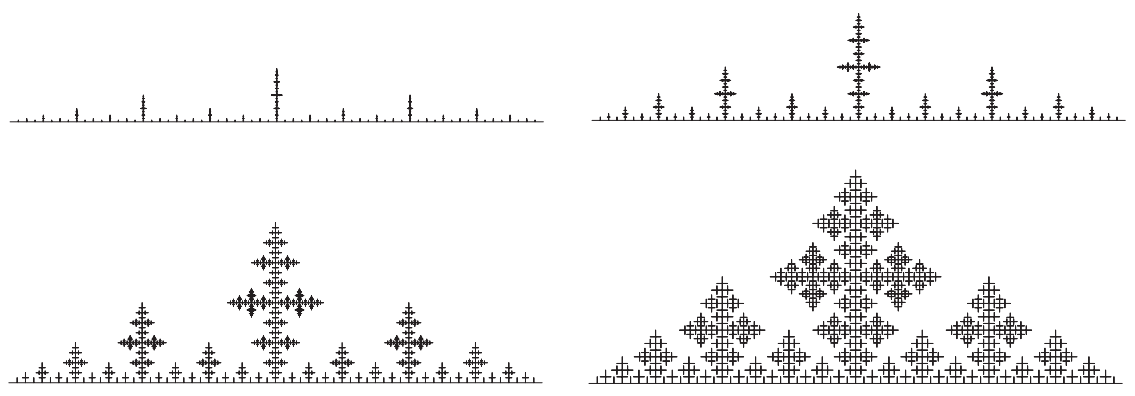

Figure 1. The antenna set for $\alpha=.1, .2, .3, .4$

Choose some $\gamma$ with $\alpha<\gamma<\frac{1}{2}$ and consider the open triangle $T=T_{\gamma}$ with vertices $0,1, \frac{1}{2}+i \gamma$. Then $F_{\alpha}(T)$ has four disjoint components and each is contained in $T$ (see Figure 22). Hence the open set condition (page 118 of [5]) is satisfied and so $X_{\alpha}$ has Hausdorff dimension $d$ where $d$ is the unique solution to

$$
2\left(\frac{1}{2}\right)^{d}+2(\alpha)^{d}=1
$$

and will have positive and finite Hausdorff measure in this dimension (see Theorem 9.3 of [5]). Note that $\operatorname{Cdim}\left(X_{\alpha}\right) \geq 1$ (since it is a continuum) and that $d \rightarrow 1$ as $\alpha \rightarrow 0$. Thus in order to show that $X_{\alpha}$ has the desired properties, we need only verify the following two claims.

Lemma 2. For any $0<\alpha, \beta<\frac{1}{2}$, there is a quasiconformal $f$ with $f\left(X_{\alpha}\right)=X_{\beta}$.

Lemma 3. For any $0<\alpha<\frac{1}{2}$ and any quasisymmetric map $f, \operatorname{dim}\left(f\left(X_{\alpha}\right)\right)>1$.

Proof of Lemma 2. The proof of this is little more than a picture which describes how to build $f$. Choose $\gamma$ so that $\max (\alpha, \beta)<\gamma<\frac{1}{2}$ and as above let $T=T_{\gamma}$ be the triangle with vertices 0,1 and $\frac{1}{2}+i \gamma$. Let $P_{\alpha}=T \backslash F_{\alpha}(T)$ and $P_{\beta}=T \backslash F_{\beta}(T)$. See Figure 2. $T$ can clearly be mapped to itself by a mapping $g$ which is the identity on the boundary, is linear and conformal on each component of $F_{\alpha}(T)$ and which is piecewise affine from $P_{\alpha}$ to $P_{\beta}$. Define $f$ to be the identity outside $T$ and define
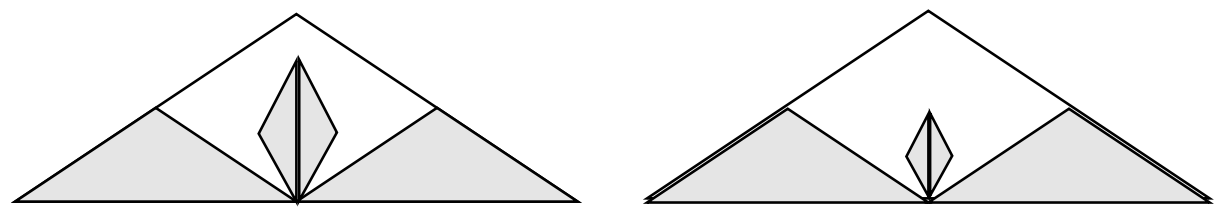

Figure 2. Building the map from $X_{\alpha}$ to $X_{\beta}$ 
it in $T \backslash X_{\alpha}$ using

$$
f(z)=f_{i_{n}}^{\beta} \circ \cdots \circ f_{i_{1}}^{\beta} \circ g \circ\left(f_{i_{1}}^{\alpha}\right)^{-1} \circ \cdots \circ\left(f_{i_{n}}^{\alpha}\right)^{-1}(z),
$$

if $z \in f_{i_{n}}^{\alpha} \circ \cdots \circ f_{i_{n}}^{\alpha}\left(P_{\alpha}\right)$, where $i_{1}, \ldots, i_{n} \in\{1,2,3,4\}$ and $f_{1}^{\alpha}=f_{1}, f_{2}^{\alpha}=f_{2}$. Then $f$ is clearly quasiconformal on $\Omega=\mathbb{R}^{2} \backslash X_{\alpha}$ and is a homeomorphism of the whole plane. By Corollary 1 of [8], $f$ is quasiconformal on the whole plane (since the complement of $X_{\alpha}$ is clearly a John domain).

Proof of Lemma [3. Since all the antenna sets are quasiconformally equivalent to one another, it is enough to prove the lemma for one of them, so we fix $\alpha=\frac{1}{4}$ and let $X=X_{1 / 4}$. Suppose $f: X \rightarrow Y$ is quasisymmetric and let $\eta$ be the corresponding homeomorphism of $[0, \infty)$. In order to show that $\operatorname{dim}(f(X))>1$, we need only construct a positive measure $\nu$ on $f(X)$ such that

$$
\nu(B(y, r)) \leq C r^{s}, \quad \forall y \in Y, \forall r>0,
$$

for some $C<\infty$ and $s>1$ (independent of $y$ and $r$ ). Then the mass distribution principle shows that for any covering of $f(X)$ by balls $B\left(y_{j}, r_{j}\right)$, we have

$$
0<\nu(f(X)) \leq \sum_{j} \nu\left(B\left(y_{j}, r_{j}\right)\right) \leq C \sum r_{j}^{s}
$$

which implies that $\operatorname{dim}(f(X)) \geq s>1$.

Let $I=[0,1] \subset X$. For any string $\sigma=\left(i_{1}, \ldots, i_{n}\right) \in\{1,2,3,4\}^{n}$, let $\sigma^{*}=$ $\left(i_{1}, \ldots, i_{n-1}\right)$. Moreover, define

$$
\begin{gathered}
X_{\sigma}=f_{i_{1}} \circ \cdots \circ f_{i_{n}}(X), \\
I_{\sigma}=f_{i_{1}} \circ \cdots \circ f_{i_{n}}(I), \\
x_{\sigma}=f_{i_{1}} \circ \cdots \circ f_{i_{n}}(0), \quad y_{\sigma}=f_{i_{1}} \circ \cdots \circ f_{i_{n}}(1), \\
d_{\sigma}=\left|f\left(x_{\sigma}\right)-f\left(y_{\sigma}\right)\right| .
\end{gathered}
$$

The first step is to show

Lemma 4. There is $s>1$ (depending only on $\eta$ ) so that $\sum_{\sigma: \sigma^{*}=\tau} d_{\sigma}^{s} \geq d_{\tau}^{s}$.

Proof. Since $x_{\tau}=x_{\tau 1}$ and $y_{\tau}=y_{\tau 2}$, the triangle inequality implies that $d_{\tau 1}+d_{\tau 2} \geq$ $d_{\tau}$ and by quasisymmetry $d_{\tau 3} \simeq d_{\tau 1}$. Thus

$$
\sum_{\sigma: \sigma^{*}=\tau} d_{\sigma} \geq\left(1+C_{1}\right) d_{\tau}
$$

for some $C_{1}>0$ depending only on $\eta$. Thus

$$
\sum_{\sigma: \sigma^{*}=\tau} \frac{d_{\sigma}}{d_{\tau}} \geq 1+C_{1}
$$

Since this is a finite sum, there exists $s>1$, depending only on $C_{1}$ and the number of terms in the sum, such that

$$
\sum_{\sigma: \sigma^{*}=\tau}\left(\frac{d_{\sigma}}{d_{\tau}}\right)^{s} \geq 1
$$

which is the desired inequality.

Fix $s>1$ as in the previous lemma. To define a measure $\mu$ on $X$ it suffices to define the measure of each $X_{\sigma}$. We let $\mu(X)=1$ and in general

$$
\mu\left(X_{\sigma}\right)=\mu\left(X_{\sigma^{*}}\right) \frac{d_{\sigma}^{s}}{\sum_{\tau: \tau^{*}=\sigma^{*}} d_{\tau}^{s}} .
$$


Lemma 5. There exist $C_{2}, C_{3}<\infty$, depending only on $\eta$ and $\operatorname{diam}(f(X))$, so that the following holds: for every $\sigma$, we have

$$
\frac{1}{C_{3}} \operatorname{diam}\left(f\left(X_{\sigma}\right)\right)^{s} \leq \frac{1}{C_{2}} d_{\sigma}^{s} \leq \mu\left(X_{\sigma}\right) \leq C_{2} d_{\sigma}^{s} \leq C_{3} \operatorname{diam}\left(f\left(X_{\sigma}\right)\right)^{s} .
$$

Proof. This is easy by induction. The central estimates hold for $X_{\sigma}=X$ with

$$
C_{2}=\max \left\{|f(0)-f(1)|,|f(0)-f(1)|^{-1}\right\}^{s} \simeq \max \left\{\operatorname{diam}(f(X)), \operatorname{diam}(f(X))^{-1}\right\}^{s}
$$

by quasisymmetry, since $\mu$ is a probability measure. In general,

$$
\mu\left(X_{\sigma}\right)=\mu\left(X_{\sigma^{*}}\right) \frac{d_{\sigma}^{s}}{\sum_{\tau: \tau^{*}=\sigma^{*}} d_{\tau}^{s}} \simeq d_{\sigma^{*}}^{s} \frac{d_{\sigma}^{s}}{\sum_{\tau: \tau^{*}=\sigma^{*}} d_{\tau}^{s}} \simeq d_{\sigma}^{s},
$$

where the estimate $\sum_{\tau: \tau^{*}=\sigma^{*}} d_{\tau}^{s} \simeq d_{\sigma^{*}}^{s}$ follows from quasisymmetry. This establishes the central two inequalities. Finally, the estimate $d_{\sigma} \simeq \operatorname{diam}\left(f\left(X_{\sigma}\right)\right)$ also follows from quasisymmetry.

Let $\nu$ be the push forward of $\mu$ to $Y$ via $f$, i.e., $\nu(E)=\mu\left(f^{-1}(E)\right)$. Now we prove (11) for the measure $\nu$. Let $B=B(y, r)$ be any ball in $Y$. We may assume $B \cap f(X) \neq \emptyset$ (or else the estimate is trivial). Furthermore, if $B$ hits $f(X)$, then it is contained in a ball of radius $2 r$ centered at a point of $f(X)$, so we may assume $y \in f(X)$. Let $x=f^{-1}(y)$ and $E=f^{-1}(B(y, r))$. By quasisymmetry, there are balls $B\left(x, r_{1}\right) \subset E \subset B\left(x, r_{2}\right)$, with the ratio $r_{2} / r_{1}$ bounded only depending on $\eta$.

Lemma 6. There are constants $C_{4}, C_{5}<\infty$ (depending only on $\eta$ ) so that $E$ can be covered by $N \leq C_{4}$ sets of the form $\left\{X_{\sigma_{j}}\right\}_{j=1}^{N}$, each of which has diameter $\leq r_{1}$ and so that $d_{\sigma_{j}} \leq C_{5} d_{\sigma_{k}}$ for any choice of $j, k \in\{1, \ldots, N\}$.

If we can prove Lemma [6] then (1) follows because

$$
\begin{aligned}
\nu(B(y, r)) & =\mu(E) \\
& \leq \sum_{j=1}^{N} \mu\left(X_{\sigma_{j}}\right) \\
& \leq C_{4} C_{5} \mu\left(X_{\sigma_{1}}\right) \\
& \leq C_{3} C_{4} C_{5} \operatorname{diam}\left(f\left(X_{\sigma_{1}}\right)\right)^{s} .
\end{aligned}
$$

If $\sigma_{1}$ is chosen so that $x \in X_{\sigma_{1}}$, then $\operatorname{diam}\left(X_{\sigma_{1}}\right) \leq r_{1}$ implies $X_{\sigma_{1}} \subset B\left(x, r_{1}\right) \subset E$, and thus $\operatorname{diam}\left(f\left(X_{\sigma_{1}}\right)\right) \leq \operatorname{diam}(f(E))=2 r$. This completes the proof of (11) except for the proof of Lemma 6 which we now give.

Proof of Lemma 6 . We shall need $X$ to be quasi-convex, i.e., that there is $C_{6}<\infty$ so that any two points $x, y \in X$ can be connected by a path in $X$ of length at most $C_{6}|x-y|$. The proof of this is left to the reader.

Given $E$, let $\left\{X_{\sigma_{j}}\right\}$ be all the sets of the form $X_{\sigma}$ with diameter $\leq r_{1}$ and which are maximal with respect to inclusion. Because the sets are nested, such a maximal collection is disjoint in the sense that their convex hulls have disjoint interiors. Moreover, each such set has diameter $\geq r_{1} / 4$ (or else its parent would be in the collection which contradicts maximality). Thus each of the disjoint hulls described above has area $\geq r_{1}^{2} / 128$. Every such set which hits $E$ is contained in $B\left(x, r_{1}+r_{2}\right) \subset B\left(x, 2 r_{2}\right)$ and so adding areas shows $N \leq C_{4}=512 \pi\left(r_{2} / r_{1}\right)^{2}$. 
To prove the second claim, note that any two of our sets $X_{\sigma_{1}}, X_{\sigma_{2}}$ which hit $E$ are at most distance $2 r_{2}$ apart and hence by the quasi-convexity of $X$ can be joined by a path $\gamma$ of length at most $2 C_{6} r_{2}$ in $X$. Assume $\gamma$ is chosen as short as possible. Then it has the property that if it hits a set $X_{\sigma}$ which does not contain the endpoints of $\gamma$, then $\gamma \cap X_{\sigma}$ is either a single point or is $I_{\sigma}$ (otherwise we can shorten $\gamma)$. Thus $X_{\sigma_{1}}$ and $X_{\sigma_{2}}$ can be joined by a chain of intervals of the form $\left\{I_{\sigma}\right\}$ where each of these has diameter between $r_{1} / 4$ and $r_{1}$ and the number of elements in the chain is at most the length of $\gamma$ divided by $r_{1} / 4$, and hence is bounded depending only on $\eta$. By quasisymmetry the $f$-images of any two adjacent intervals in the chain have comparable diameters depending only on $\eta$, and hence the same is true of $X_{\sigma_{1}}$ and $X_{\sigma_{2}}$. This completes the proof of the lemma and hence the proof of Theorem 1

Remark 1. If we are only interested in quasiconformal maps $f: \mathbb{R}^{2} \rightarrow \mathbb{R}^{2}$, then the proof of Lemma 3 can be considerably shortened using a result from [2. It is proven there that compact, connected sets which are uniformly wiggly (i.e., deviate from a line segment uniformly at all points and all scales; see [2] for the precise definition) must have dimension strictly larger than 1 . Using the compactness of $K$-quasiconformal maps, it is easy to check that any quasiconformal image of the antenna set is uniformly wiggly and hence has dimension $>1$. This proof does not apply directly to metric spaces since we don't know what "line segment" should mean in this setting. However, the proof given in this paper suggests the following possibility: $Y$ is uniformly wiggly if there is a $\beta>0$ so that for every $y \in Y$ and $r>0$, and for any collection $\left\{y_{0}, \ldots, y_{n}\right\} \subset B(y, r)$, we have

$$
\sum\left|y_{k}-y_{k-1}\right|+\sup _{z \in B(y, r)} \inf _{k}\left|z-y_{k}\right| \geq(1+\beta)\left|y_{0}-y_{n}\right| .
$$

In Euclidean space this is equivalent to the definition from [2]. Does this imply $\operatorname{dim}(Y)>1$ if $Y$ is a compact, connected metric space?

Remark 2. The construction could be modified to give an example which is a Jordan arc. On the other hand, the infimum in the definition of conformal dimension can be attained for some arcs (e.g., any quasi-arc). Thus topology alone cannot ensure the infimum in the definition of conformal dimension is attained.

Remark 3. Given a set $E \subset \mathbb{R}^{2}$ we can define $D_{E}$ to be the set of values of $\operatorname{dim}(f(E))$ as $f$ ranges over all quasiconformal maps of the plane. This must be a single point or an interval since any quasiconformal map can be connected to the identity by a path of maps along which the dimension changes continuously. From [1] and [6], we know that the only possibilities for $D_{E}$ are of the form $\{0\},\{2\}$, $(0,2),[\alpha, 2)$ and $(\alpha, 2)$ for $0<\alpha<2$. The first three are well known to occur, as is $[1,2)$. This paper shows that $(1,2)$ can also occur. The second author showed in [10] that intervals of the form $[\alpha, 2)$ can occur if $1<\alpha<2$ and conjectured that $[\alpha, 2),(\alpha, 2)$ never occur for $0<\alpha<1$. Do the intervals $(\alpha, 2)$ occur for $1<\alpha<2: 1$

Remark 4. Computing the conformal dimension seems difficult, even for some fairly "simple" fractal sets. For example, what is the conformal dimension of the Sierpinski carpet illustrated in Figure 3. Its dimension is $\log 8 / \log 3 \approx 1.89279$ and its

\footnotetext{
${ }^{1}$ After this paper was completed, we answered this question in the affirmative; see [3].
} 
conformal dimension is $\geq 1+\log 2 / \log 3 \approx 1.63093$ (since it contains the Cartesian product of the middle thirds Cantor set with an interval, for which the results of 10] imply Cdim = dim).

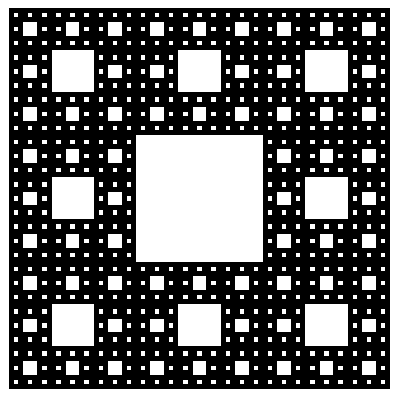

FIGURE 3. The Sierpinski carpet

\section{REFERENCES}

1. C. J. Bishop, Quasiconformal mappings which increase dimension, Ann. Acad. Sci. Fenn. Ser. A I Math. 24 (1999), 397-407. MR 2000i:30044

2. C. J. Bishop and P. W. Jones, Wiggly sets and limit sets, Ark. Mat. 35 (1997), no. 2, 201-224. MR 99f:30066

3. C. J. Bishop and J. T. Tyson, Locally minimal sets for conformal dimension, Ann. Acad. Sci. Fenn. Ser. A I Math. (to appear).

4. M. Bourdon, Au bord de certains polyèdres hyperboliques, Ann. Inst. Fourier (Grenoble) 45 (1995), 119-141. MR 96b:20045

5. K. J. Falconer, Fractal geometry, Mathematical Foundations and Applications, John Wiley and Sons Ltd., Chichester, 1990. MR 92j:28008

6. F. W. Gehring and J. Väisälä, Hausdorff dimension and quasiconformal mappings, J. London Math. Soc. (2) 6 (1973), 504-512.

7. J. Heinonen, Lectures on analysis on metric spaces, Univ. of Michigan (1996), Lecture notes.

8. P. W. Jones, On removable sets for Sobolev spaces in the plane, Essays on Fourier analysis in honor of Elias M. Stein (Princeton, NJ, 1991) (Princeton University Press, Princeton, NJ), 1995, pp. 250-267.

9. P. Pansu, Dimension conforme et sphère à l'infini des variétés à courbure négative, Ann. Acad. Sci. Fenn. Ser. A I Math. 14 (1989), 177-212. MR 90k:53079

10. J. T. Tyson, Sets of minimal Hausdorff dimension for quasiconformal maps, Proc. Amer. Math. Soc. (to appear). CMP 99:09

Department of Mathematics, SUny at Stony Brook, Stony Brook, New York 117943651

E-mail address: bishop@math.sunysb.edu

Department of Mathematics, SUNy at Stony Brook, Stony Brook, New York 117943651

E-mail address: tyson@math.sunysb.edu 